\title{
Is Routine Brain CT Scan, Performed for Early Follow Up in Head Trauma Patients with GCS 14-15, Always Justified?
}

\author{
Kessel Boris ${ }^{1 *}$, Itamar Ashkenazi ${ }^{2}$, Zeina Abdel Rauf ${ }^{3}$, Nachtigal Alicia ${ }^{3}$, Korin Alexander ${ }^{1}$, Khashan T RN $^{1}$ and Ricardo Alfici ${ }^{2}$ \\ ${ }^{1}$ Trauma Unit, Hillel Yaffe Level 2 Trauma Center, Israel \\ ${ }^{2}$ Surgical Division, Hillel Yaffe Level 2 Trauma Center, Israel \\ ${ }^{3}$ Radiology Department, Hillel Yaffe Level 2 Trauma Center, Israel
}

\begin{abstract}
Hypothesis: Routine repeat head Computed Tomography (CT) for patients with traumatic head injury, initially presenting with GCS of 14-15, does not change therapeutic policy in these trauma patients.

Methods: This was a retrospective cohort study of trauma patients with Glasgow Coma Scale (GCS) of 14-15 on admission, suffering from different types of intracranial bleeding who were admitted for observation in a level II trauma center. The size of hematoma on initial head CT was measured and compared to findings of repeat CT performed following 12 hours. Patients were evaluated as to changes in neurologic status and treatment.
\end{abstract}

Results: 68 patients treated over a period of 5 years were evaluated. Forty two (61.8\%) were male and 24 $(38.2 \%)$ were female. Mean age was 56.2 years and mean ISS score was $12 \pm 5.1$. Initial GCS was 15 in 51 patients and 14 in 15 other patients. CT scan revealed 7 epidural hematomas, 20 subdural hematomas, twenty eight intraparenchymal bleeding, and 13 subarchnoid hemorrhages. Repeat CT revealed an increase in size of the hematoma in 8 eight patients. None of these patients suffered from clinical deterioration. Repeat CT in 12 patients who's GCS deteriorated, did not show any significant changes on the repeat CT scan. None of the patients underwent intervention after a routine repeat $\mathrm{CT}$.

Conclusions: Routine repeat CT scan of head, performed 12 hours after the initial scan did not change the therapeutic policy in GCS 14-15 head trauma patients. The need for mandatory CT of head in mild traumatic brain injury should be questioned.

Keywords: Head trauma; Intracranial bleeding; Epidural hematoma; Subdural hematoma; CT scan; Follow up

Abbreviations: TBI: Traumatic Brain Injury; MHI: Minimal Head Injury; ICH: Intra Cranial Hemorrhage; CT: Computerized Tomography; RCT: Repeat Computerized tomography; GCS: Glasgow Coma Scale; NE: Neurological Examination; ICP: Intra Cranial Pressure; PPV: Positive Predictive Value; NPV: Negative Predictive Value

\section{Introduction}

Mild Traumatic Brain Injury (TBI) is common, with an estimated incidence between 100-300 cases per 100,000 people in the Western world [1]. Over 1.7 million Americans present to emergency departments with TBI where, overall, in the United States alone, it results in 52000 deaths and 275000 hospital admissions annually and is a contributing factor in one-third of all injury-related deaths [2]. Of all TBI patients, $>80 \%$ of cases could be classified as Minimal Head Injuries, (MHI) where there may or may not be loss of consciousness and/or post-traumatic retrograde amnesia but where the presenting Glasgow Coma Scale (GCS) exceeds 12 [3,4]. When patients presenting with MHI have an Intracranial Hemorrhage (ICH) detected by initial head CT, a standard of care in many institutions is to obtain a second follow-up CT scan within 24 hours (a repeat CT-RCT) in order to define the need for neurosurgical intervention or patient transfer to a neurosurgical unit. These decisions have hitherto largely been made independently of the patient's clinical neurological status. This protocol has been designed to rule out progression of an intracranial bleed and to evaluate the manageable aspects of secondary brain injury. Although the performance of an RCT in MHI with attendant ICH is well established, it has been little tested [5]. Assessment of the clinical value of this protocol will assist in the timely transfer of high risk cases and will better direct the discharge of TBI patients as well as reduce hospital costs and diminish unwarranted radiation exposure [6-8].

Guidelines have been published for the management of moderate TBI, [9] where it is recognized that accurate, more widely available neuroimaging is an integral tool for patients in the diagnosis of lifethreatening injuries as well as to define prognosis and follow-up. There are both retrospective and prospective studies which have examined the clinical benefit of RCT in this setting, where most of the available literature has shown little advantage for those patients where the Neurological Examination (NE) is normal on admission [10-16]. By contrast, nearly one-quarter of patients with MHI are clinically neurologically abnormal on admission [10], although Sifri et al. have recently reported that no patients with a persistently abnormal NE had a clinically beneficial RCT which changed management [16]. In their study of 107 patients, only 6 patients (6.5\%) with an acutely deteriorating NE underwent an RCT which actually directed a change of management. The aims of this study were to evaluate the clinical value of RCT in patients initially presenting with an MHI, (presentation GCS

*Corresponding author: Boris Kessel MD, Trauma Unit, Hillel Yaffe Medical Center, POB 169, Hadera, Israel 38100, Tel: 972-4-6304407; Fax: 972-4-6304545; E-mail: blko2@yahoo.com

Received May 28, 2013; Accepted July 29, 2013; Published July 31, 2013

Citation: Boris K, Ashkenazi I, Rauf ZA, Alicia N, Alexander K, et al. (2013) Is Routine Brain CT Scan, Performed for Early Follow Up in Head Trauma Patients with GCS 14-15, Always Justified? J Trauma Treat 2: 174. doi:10.4172/2167 1222.1000174

Copyright: (C) 2013 Boris K, et al. This is an open-access article distributed unde the terms of the Creative Commons Attribution License, which permits unrestricted use, distribution, and reproduction in any medium, provided the original author and source are credited. 
14 or 15), where initial CT scan confirmed an ICH and where patients presented to the emergency room with or without an abnormal NE.

\section{Materials and Methods}

Hillel Yaffe Medical Center (HYMC) is a level II trauma center without neurosurgical capacity.

According to the trauma unit's protocol, all positive head CT scans are evaluated by the on-call radiologist and via Picture Archiving and Communication System (PACS) and reviewed by an on call neurosurgeon stationed in a level I trauma center. Once a joint decision has been made to hospitalize a patient for observation in HYMC, a repeat CT is routinely performed following an 12 hour interval if not done beforehand because of neurologic deterioration.

This study was authorized by the HYMC's Institutional Review Board.

All patients with blunt traumatic Mild Head Injuries (MHI) admitted to our trauma center were reviewed and were the subject of this analysis. Patients were identified by cross-checking of hospital trauma and radiology databases with approval for this retrospective assessment of the medical records of these patents and their radiology being obtained from the local hospital Institutional Review Board. Study inclusion criteria were: an admission GCS of 14 or 15 and a positive initial CT scan with a finding of ICH including subdural hematoma, epidural hematoma, intraparenchymal hematoma and subarachnoid hemorrhage. Every enrolled patient underwent a repeat CT (RCT) within 12 hours of the initial CT scan or depending on clinical neurological deterioration. Patients without full data or an RCT and were excluded from analysis as were patients who were transferred based on the initial CT scan and neurosurgical consult. The principal outcome measure was to evaluate the frequency of change in hematoma size between two CT scans in GCS 14-15 head injured patients and the relations of changes in $\mathrm{CT}$ to changes in neurologic status.

Clinical deterioration on NE included any decrease in the level of consciousness, any new motor or sensory deficit, or symptoms suggestive of raised ICP, (persistent severe headache and/or vomiting). General patient demographics were recorded, (age, gender, ISS score on admission, admission GCS, follow-up GCS, type of ICH, measured dimensions of ICH, out of protocol indications for RCT, changes in neurologic status and the need for management changes and/ or neurosurgical intervention). In analysis, categorical variables are reported as proportions $(+95 \% \mathrm{CI})$ where appropriate and continuous variables are presented as means $( \pm \mathrm{SD})$.

\section{Results}

During period of five years, sixty eight patients treated between January 1, 2007 to December 31, 2011, were included to this study. Forty two (61.8\%) were male and $26(38.2 \%)$ were female. Mean age was 56.2 years with range of 39 to 36.2 years. The overall mean ISS score was (ISS) was $14.9 \pm 5.1$. Initial GCS was 15 in 51 patients.

Of the total number of cases, 28 patients had intraparenchymal hemorrhage on the admission CT scan. In this group, the mean ISS was $13.7 \pm 6$. (Table 1 shows 28 patients with intraparenchymal hemorrhage). In only one patient did the dimensions of the contusion increase on RCT, with 8 patients showing slight reduction in their follow-up GCS. (Table 1)

Table 2 shows 7 patients presenting with epidural hematomas. In this group, the mean ISS was $17.6 \pm 3.7$. One patient showed enlargement of the measurable dimensions of the hematoma on RCT and no patient showed GCS deterioration during follow-up.

Table 3 shows 20 patients presenting with subdural hematomas. In this group, the mean ISS was $15.3 \pm 4$. Three patients showed enlargement of the measurable dimensions of the hematoma on RCT with none of these 3 showing GCS deterioration during follow-up. Four other patients showed minor GCS deterioration during follow-up examination.

Table 4 shows 13 patients presenting with subarachnoid hemorrhage. In this group, the mean ISS was $15 \pm 2.2$. Three patients showed enlargement of the measurable dimensions of the hemorrhagic area on RCT with none of these 3 (and no other patient in this group) showing GCS deterioration during follow-up. Overall, there was progression of the extent of intracranial hemorrhage in $8(15 \%)$ cases.

There was no correlation between any changes in the GCS and variations in the extent of hemorrhage $(r=-0.173)$. Of the total patient group, there was mild clinical GCS deterioration in 12 cases where there was no alteration on RCT in hemorrhagic extent. By contrast, 8 patients showed enlargement on RCT of their hemorrhage although none had clinical deterioration of their NE. Of the total, 4 patients received therapeutic changes during the follow-up period. In one case with a subdural hematoma, the GCS reduced from 14 to 12 , prompting an earlier RCT whereas in another case with a subarachnoid hemorrhage, expansion of the hemorrhagic area resulted in transfer to a neurosurgical unit without definitive change in the neurologic status or outcome. Two other patients, (both with extradural hematomas and coincident contusion) were also transferred as part of a departmental protocol for this type of $\mathrm{ICH}$. In both cases, there was no change in

\begin{tabular}{|c|c|c|c|}
\hline $\begin{array}{c}\text { Intraparenchimal } \\
\text { Hemorrhage }\end{array}$ & $\begin{array}{c}\text { No. patients (\% } \\
\text { from subgroup) }\end{array}$ & $\begin{array}{c}\text { No. patients } \\
\text { deterioration of } \\
\text { GCS }\end{array}$ & $\begin{array}{c}\text { No. patients } \\
\text { transferred to } \\
\text { Neurosurgery }\end{array}$ \\
\hline Increase in size & $1(3 \%)$ & 0 & 0 \\
\hline No change & $10(36 \%)$ & 2 & 1 \\
\hline Decrease in size & $17(61 \%)$ & 1 & 0 \\
\hline
\end{tabular}

Table 1: 28 patients with intraparenchimal hemorrhage.

\begin{tabular}{|c|c|c|c|}
\hline $\begin{array}{c}\text { Epidural } \\
\text { Hemorrhage }\end{array}$ & $\begin{array}{c}\text { No. patients (\% } \\
\text { from subgroup) }\end{array}$ & $\begin{array}{c}\text { No. patients } \\
\text { deterioration of } \\
\text { GCS }\end{array}$ & $\begin{array}{c}\text { No. patients transferred } \\
\text { to Neurosurgery }\end{array}$ \\
\hline Increase in size & $1(14 \%)$ & 0 & 0 \\
\hline No change & $2(29 \%)$ & 0 & 1 \\
\hline Decrease in size & $4(57 \%)$ & 0 & 0 \\
\hline
\end{tabular}

Table 2: 7 patients presenting with epidural hematom.

\begin{tabular}{|c|c|c|c|}
\hline $\begin{array}{c}\text { Subdural } \\
\text { Hemorrhage }\end{array}$ & $\begin{array}{c}\text { No. patients (\% } \\
\text { from subgroup) }\end{array}$ & $\begin{array}{c}\text { No. patients } \\
\text { deterioration of } \\
\text { GCS }\end{array}$ & $\begin{array}{c}\text { No. patients } \\
\text { transferred to } \\
\text { Neurosurgery }\end{array}$ \\
\hline Increase in size & $3(15 \%)$ & 0 & 0 \\
\hline No change & $1(5 \%)$ & 2 & 0 \\
\hline Decrease in size & $16(80 \%)$ & 2 & 1 \\
\hline
\end{tabular}

Table 3: 20 patients presenting with subdural hematomas.

\begin{tabular}{|c|c|c|c|}
\hline $\begin{array}{c}\text { Subarachnoid } \\
\text { Hemorrhage }\end{array}$ & $\begin{array}{c}\text { No. patients (\% } \\
\text { from subgroup) }\end{array}$ & $\begin{array}{c}\text { No. patients } \\
\text { deterioration of } \\
\text { GCS }\end{array}$ & $\begin{array}{c}\text { No. patients } \\
\text { transferred to } \\
\text { Neurosurgery }\end{array}$ \\
\hline Increase in size & $3(23 \%)$ & 0 & 1 \\
\hline No change & $3(23 \%)$ & 2 & 0 \\
\hline Decrease in size & $7(54 \%)$ & 3 & 0 \\
\hline
\end{tabular}

Table 4: 13 patients presenting with subarachnoid hemorrha. 
hemorrhagic extent on RCT or in the GCS on NE. None of these 4 patients required neurosurgical intervention.

\section{Discussion}

The importance in clinical management of the initial head CT scan in those patients presenting with Minimal Head Injury (MHI) is well established [10,17], however, there is no real consensus regarding the clinical utility of Repeat CT (RCT) in this patient population. Our data would suggest that patients with MHI and ICH who present with a normal Neurological Examination (NE) on admission without acute deterioration during follow-up, do not require an RCT and have no need for neurosurgical intervention regardless of the type of intracranial bleeding. There was from our small study, no clear correlation between changes in the extent of hemorrhage on RCT and neurological status, although it is accepted that the admission GCS criteria of the study results in a bias towards less severe injuries and a non-interventional outcome.

Our data where patients present with an essentially normal NE, (with a MHI and a demonstrated ICH), are in agreement with that previously reported. $[11,12]$. The literature concerning the usefulness of an RCT in similar patients, but where there is an abnormal presenting NE, is more complicated where neurosurgical intervention is required in $6 \%$ of such cases but only in those where there is an acute neurological deterioration [16]. Patients with a persistently abnormal NE (without deterioration from a baseline abnormality), in this study by Sifri et al. [16] did not benefit from a routine RCT. Our data showed no correlation in any type of hemorrhage (or its evolution) with deterioration in the neurological status. This finding is also in agreement with that of Velmahos et al. [14] who reported the association between neurosurgical intervention and acute clinical neurologic deterioration, where RCT performed within a mean of 13 hours after admission showed a worsening of the brain lesion in $21 \%$ of cases. In this study, in the few patients where there was neurosurgical intervention ( 2 craniotomies and hematoma evacuations and 2 placements of an ICP monitor), the clinical deterioration preceded the RCT in each case. Similar findings have also been reported by Brown et al. [15] where changes in NE predated investigation and intervention in patients presenting with traumatic brain injury, regardless of injury severity.

These findings have also been emphasized in a recent systematic review of the available literature by Stippler et al. [18]. In this review, routine RCT resulted in a neurosurgical intervention rate in only $0.7 \%$ of cases (11/1574 patients) compared with an intervention rate of $42.9 \%$ (24/56 patients) where there was an acute clinical neurological deterioration. The policy of any unit towards invasive ICP monitoring will define the intervention rate and a more liberal approach towards placement of ICP monitors will obviate the value of routine RCT in such patients. The findings for patients with MHI also reflect similar reports which have defined the utility of RCT in moderate and severe traumatic brain injury $[19,20]$. The implication from this data is that significant $\mathrm{ICH}$, even if changing in the absence of a change in $\mathrm{NE}$ does not require further imaging or neurosurgical transfer. This finding is supported by the data from Sifri et al. [16] who showed a Negative Predictive Value (NPV) for neurosurgical intervention of a serially non-deteriorating NE of $100 \%$ where conversely, the Positive Predictive Value (PPV) of NE and RCT for intervention were both very low (29\% and $9.6 \%$, respectively).

There are several limitations to our study and its interpretation based around its small group size, its retrospective design, the biases of patient inclusion and exclusion criteria and the lack of standardization and blinding of both the initial and the follow-up CT examinations. The inclusion of MHI is a bias towards non-intervention and reduces the potential clinical value of RCT when compared with those patients presenting with more severe TBI. In this respect, there is greater consensus in what is agreed to be a normal NE, however, better standardization of the presently subjective criteria representing acute neurological deterioration is still required [13]. It may be that further subpopulation analysis will define specialized risk groups where Kaups et al. have shown in severe brain injury that RCT alone will generally not direct neurosurgical intervention [21]. Here, other factors such as the presence of an underlying coagulopathy [22], older age [14, 23 ] and an initial multiplicity of intracranial lesions could potentially identify patients at higher risk for neurosurgical treatment. In order to answer this question, larger studies are required with prospective randomization of patients to either routine or selective clinically-based RCT which compare the PPV and the NPV for serial NE and/or RCT with the need for neurosurgical intervention. Equally, the known natural history of MHI will influence the clinical value and timing of an RCT where it has been shown that two-thirds of those cases which deteriorate do so within 6 hours of admission [16] and where almost all cases of ICH cease progression by 48 hours [24]. Further research is needed concerning the timing of RCT in MHI where it potentially could be used as a factor which might unmask radiologic progression prior to clinical deterioration.

The clinical recommendation not to perform routine RCT in these cases, but rather to closely monitor and document neurologic status, has important implications, reducing the risks associated with patient transport, [6] the patient radiation exposure, [8] hospital costs, the overall length of hospital stay, the length of ICU stay and the impact on delays for other patients scheduled for CT examinations. For those patients undergoing transfer, the risks of complications are considerable; the most common of which are hemodynamic instability, raised ICP, desaturation and agitation [19]. The incorporation within larger analyses of different types of ICH will also better define their natural history and the need for further early neuro imaging [25]. Here, it is intuitive that patients with an epidural hematoma or those with a skull fracture traversing the middle meningeal artery territory or that of a major venous sinus should benefit from an RCT, where there is a lower threshold for surgical intervention. The small number of such cases in our study did not permit sufficient separate analysis of this group where hemorrhagic enlargement has been previously reported in the face of clinical stability [26]. The site of ICH will also influence the predicted timing of both an RCT and of neurological decline, where Fainardi et al. have shown that the full extent of a post-traumatic subarachnoid hemorrhage is often missed on the first CT scan and that classification of its severity takes time to evolve [27].

In this respect, Velmahos et al. have shown that a short interval ( $<90$ minutes) between admission and the initial head CT was an independent predictor of a worsening finding on RCT [14]. Knowledge concerning the differential evolution times to the final hematoma size will permit better definition of appropriate observation periods in patients with mild complicated traumatic brain injury if a policy for routine RCT in such cases is abandoned [28]. This approach will remain controversial, where Bee et al. have published data with contradictory recommendations, somewhat dependent upon the initial type of $\mathrm{ICH}$ and where 5/207 patients in their study admitted with $\mathrm{MHI}$ and $\mathrm{ICH}$, (all with subdural hematomas), underwent craniotomies which were clinically silent but where there was radiological progression [29].

In summary, the clinical utility of a routine early RCT in patients presenting with $\mathrm{MHI}$ and $\mathrm{ICH}$ is unproven, where close serial NE 
acts as the main predictive factor for repeat neuroimaging and for neurosurgical intervention. Improved understanding of the risk factors for neurological decline in these patients will direct appropriate neurosurgical consultation [30] and is likely to be more cost-effective, where the economic benefit of RCT in MHI cases diminishes with increasing patient age [31]. The decisions made for routine postadmission neuroimaging in MHI have important medicolegal significance, where RCT is considered a standard care even when it may not be medically indicated and where presently, such a recommendation is still viewed with caution. These recommendations will not influence the use of routine RCT in patients with more severe TBI where regular neuroimaging is needed in intubated patients and those with a low GCS and where even ICP monitoring may miss an evolving focal process requiring a craniotomy. As a follow-up (or derivative) test, the use of an RCT will also be affected by over- or underuse of initial CT scanning in the emergency room in patients presenting with a minor head injury [32].

\section{Conclusions}

Repeat CT of most head injured patients with GCS of 14-15, suffering from intracranial traumatic lesion, will not demonstrate significant changes in the size of intracranial hematoma.

Increase in hematoma size is not prognostic factor of neurological deterioration.

Routine repeat CT scan may not be justified in head trauma patients with GCS $14-15$ on admission.

Large prospective studies are needed to consolidate these conclusions.

\section{References}

1. Cassidy JD, Carroll LJ, Peloso PM (2004) Incidence, risk factors and prevention of mild traumatic brain injury: results of the WHO Collaborating Centre Task Force on Mild Traumatic Brain Injury. J Rehab Med: 43 (Suppl): 28-60

2. Coronado VG, Xu L, Basavaraju SV, McGuire LC, Wald MM, et al. (2011) Surveillance for traumatic brain injury-related deaths--United States, 19972007. MMWR Surveill Summ 60: 1-32

3. Levin HS (1996) Outcome from mild head injury. Neurotrauma New York; McGraw Hill 749-54

4. Faul M, Xu L, Wald MM, Coronado VG (2010) Traumatic brain injury in the United States: Emergency department visits, hospitalizations and deaths 20022006, Atlanta, GA Centers for Disease Control and Prevention, National Center for Injury Prevention and Control.

5. Alahmadi H, Vachhrajani S, Cusimano MD (2010) The natural history of brain contusion: an analysis of radiological and clinical progression. J Neurosurg 112: 1139-1145

6. Stearley HE (1998) Patients' outcomes: intrahospital transportation and monitoring of critically ill patients by a specially trained ICU nursing staff. Am J Crit Care 7: 282-287.

7. Figg RE, Burry TS, Vander Kolk WE (2003) Clinical efficacy of serial computed tomographic scanning in severe closed head injury patients. J Trauma 55 1061-1064.

8. Brenner DJ, Hall EJ (2007) Computed tomography: an unnecessary source of radiation exposure. $\mathrm{N}$ Engl J Med 357: 2277-84.

9. Cushman JG, Agarwal N, Fabian TC, Garcia V, Nagy KK, et al. (2001) Practice management guidelines for the management of mild traumatic brain injury: the EAST practice management guidelines work group. J Trauma 51: 1016-1026.

10. Livingston DH, Loder PA, Koziol J, Hunt CD (1991) The use of CT scanning to triage patients requiring admission following minimal head injury. J Trauma 31: 483-487.

11. Sifri ZC, Livingston DH, Lavery RF, Homnick AT, Mosenthal AC, et al. (2004) Value of repeat cranial computed axial tomography scanning in patients with minimal head injury. Am J Surg 187: 338-342.
12. Sifri ZC, Homnick AT, Vaynman A, Lavery R, Liao W, et al. (2006) A prospective evaluation of the value of repeat cranial computed tomography in patients with minimal head injury and an intracranial bleed. J Trauma 61: 862-867.

13. Wang MC, Linnau KF, Tirschwell DL, Hollingworth W (2006) Utility of repea head computed tomography after blunt head trauma: a systematic review. J Trauma 61: 226-233.

14. Velmahos GC, Gervasini A, Petrovick L, Dorer DJ, Doran ME, et al. (2006) Routine repeat head CT for minimal head injury is unnecessary. J Trauma 60 494-499.

15. Brown CV, Zada G, Salim A, Inaba K, Kasotakis G, et al. (2007) Indications for routine repeat head computed tomography (CT) stratified by severity of traumatic brain injury. J Trauma 62: 1339-1344.

16. Sifri ZC, Nayak N, Homnick AT, Mohr AA, Yonclas P, et al. (2011) Utility of repeat head computed tomography in patients with an abnormal neurologic examination after minimal head injury. J Trauma 71: 1605-1610.

17. Nagy KK, Joseph KT, Krosner SM, Roberts RR, Leslie CL, et al. (1999) The utility of head computed tomography after minimal head injury. J Trauma 46 268-270.

18. Stippler M, Smith C, McLean AR, Carlson A, Morley S, et al. (2012) Utility of routine follow-up head CT scanning after mild traumatic brain injury: a systematic review of the literature. Emerg Med J 29: 528-532.

19. Lee TT, Aldana PR, Kirton OC, Green BA (1997) Follow-up computerized tomography (CT) scans in moderate and severe head injuries: correlation with Glasgow Coma Scores (GCS), and complication rate. Acta Neurochir (Wien) 139: $1042-1047$

20. Tabori U, Kornecki A, Sofer S, Constantini S, Paret G, et al. (2000) Repeat computed tomographic scan within 24-48 hours of admission in children with moderate and severe head trauma. Crit Care Med 28: 840-844.

21. Kaups KL, Davis JW, Parks SN (2004) Routinely repeated computed tomography after blunt head trauma: does it benefit patients? J Trauma 56: 475-480.

22. Kaufman HH, Moake JL, Olson JD, Miner ME, duCret RP, et al. (1980) Delayed and recurrent intracranial hematomas related to disseminated intravascular clotting and fibrinolysis in head injury. Neurosurgery 7: 445-449.

23. Reynolds FD, Dietz PA, Higgins D, Whitaker TS (2003) Time to deterioration of the elderly, anticoagulated, minor head injury patient who presents without evidence of neurologic abnormality. J Trauma 54: 492-496.

24. Homnick A, Sifri Z, Yonclas P, Mohr A, Livingston D (2012) The temporal course of intracranial haemorrhage progression: how long is observation necessary? Injury 43: 2122-2125

25. Chieregato A, Fainardi E, Morselli-Labate AM, Antonelli V, Compagnone C, et al. (2005) Factors associated with neurological outcome and lesion progression in traumatic subarachnoid hemorrhage patients. Neurosurgery 56: 671-680.

26. Sullivan TP, Jarvik JG, Cohen WA (1999) Follow-up of conservatively managed epidural hematomas: implications for timing of repeat CT. AJNR Am J Neuroradiol 20: 107-113

27. Fainardi E, Chieregato A, Antonelli V, Fagioli L, Servadei F (2004) Time course of CT evolution in traumatic subarachnoid haemorrhage: a study of 141 patients. Acta Neurochir (Wien) 146: 257-263.

28. Yamaki T, Hirakawa K, Ueguchi T, Tenjin H, Kuboyama T, et al. (1990) Chronological evaluation of acute traumatic intracerebral haematoma. Acta Neurochir (Wien) 103: 112-115

29. Bee TK, Magnotti LJ, Croce MA, Maish GO, Minard G, et al. (2009) Necessity of repeat head CT and ICU monitoring in patients with minimal brain injury. Trauma 66: 1015-1018.

30. Huynh T, Jacobs DG, Dix S, Sing RF, Miles WS, et al. (2006) Utility of neurosurgical consultation for mild traumatic brain injury. Am Surg 72: 11621165.

31. Stein SC, Fabbri A, Servadei F (2008) Routine serial computed tomographic scans in mild traumatic brain injury: when are they cost-effective? J Trauma 65: 66-72.

32. Smits M, Dippel DW, de Haan GG, Dekker HM, Vos PE, et al. (2005) Externa validation of the Canadian CT Head Rule and the New Orleans Criteria for CT scanning in patients with minor head injury. JAMA 294: 1519-1525. 